University of Wollongong

Research Online

Faculty of Engineering - Papers (Archive)

Faculty of Engineering and Information

Sciences

1999

\title{
Quantum point contact in a magnetic field: Far-infrared resonant heating observed in photoconductivity
}

\author{
R. J. Heron \\ University of Wollongong \\ R. A. Lewis \\ roger@uow.edu.au,roger@uow.edu.au \\ B. E. Kane \\ University of New South Wales \\ G. R. Facer \\ University of New South Wales \\ R. G. Clark \\ University of New South Wales
}

See next page for additional authors

Follow this and additional works at: https://ro.uow.edu.au/engpapers

Part of the Engineering Commons

https://ro.uow.edu.au/engpapers/459

\section{Recommended Citation}

Heron, R. J.; Lewis, R. A.; Kane, B. E.; Facer, G. R.; Clark, R. G.; Dzurak, A. S.; Lumpkin, N. E.; Starrett, R. P.; Rickel, D. G.; Pfeiffer, L. N.; and West, K. W.: Quantum point contact in a magnetic field: Far-infrared resonant heating observed in photoconductivity 1999.

https://ro.uow.edu.au/engpapers/459 


\section{Authors}

R. J. Heron, R. A. Lewis, B. E. Kane, G. R. Facer, R. G. Clark, A. S. Dzurak, N. E. Lumpkin, R. P. Starrett, D. G. Rickel, L. N. Pfeiffer, and K. W. West 


\title{
Quantum point contact in a magnetic field: Far-infrared resonant heating observed in photoconductivity
}

\author{
R. J. Heron and R. A. Lewis ${ }^{\text {a) }}$ \\ Department of Physics, University of Wollongong, Wollongong, New South Wales 2522, Australia \\ B. E. Kane, G. R. Facer, R. G. Clark, A. S. Dzurak, N. E. Lumpkin, and R. P. Starrett \\ Semiconductor Nanofabrication Facility, University of New South Wales, Sydney 2052, Australia \\ D. G. Rickel \\ National High Magnetic Field Laboratory, Los Alamos National Laboratory, Los Alamos, \\ New Mexico 87545 \\ L. N. Pfeiffer and K. W. West \\ Bell Laboratories, Lucent Technologies, Murray Hill, New Jersey 07974
}

(Received 1 July 1999; accepted for publication 22 September 1999)

We report on the far-infrared photoresponse of a quantum point contact device fabricated on a top-gated GaAs/AlGaAs heterostructure. The top-gated architecture avoids the disorder built into conventional modulation-doped structures. We observe a distinctive far-infrared magnetophotoresponse. This depends on the wavelength of the radiation and on the carrier density, which is controlled by the gate voltage. We conclude by comparison with transport data that the oscillations observed in photoconductivity and which are centred around the cyclotron energy arise from the resonant heating of electrons by the far-infrared radiation. (C) 1999 American Institute of Physics. [S0003-6951(99)03346-X]

Recently, quantized conductance was reported in quantum wire (QWR) and quantum point contact (QPC) devices in which the electron density and confining potential are independently controlled by lithographically defined gates. ${ }^{1}$ Conductance plateaus were observed near $G=2 N e^{2} / h$ in the QWRs and additional structure was observed for $G<2 e^{2} / h$ in the QPC. The QWRs and QPC were not based on a traditional modulation-doped heterostructure but rather on a nominally undoped enhancement-mode field effect transistor (FET) architecture, in which electrons are introduced via a conducting top gate. ${ }^{2}$ This approach avoids the intentional introduction of dopants and consequent disorder and permits the independent adjustment of carrier concentration and confinement potential by appropriate gates. The twodimensional electron gas (2DEG) developed in such FETs is of extremely high quality. This is evident in both electrical measurements, where ballistic transport exceeding $160 \mu \mathrm{m}$ has been observed, ${ }^{3}$ and in far-infrared (FIR) optical measurements, in which very narrow cyclotron resonance (CR) peaks have been observed. ${ }^{4}$ In this letter we extend the recent optical work ${ }^{4}$ on the FET 2DEG and complement the recent transport experiments ${ }^{1}$ on the FET-based QPC by reporting on the FIR reponse of a FET-based QPC device over a range of carrier densities and magnetic field strengths.

Evidence of quantized conductance was first reported in QPCs defined electrostatically using surface Schottky gates on GaAs/AlGaAs heterojunctions. ${ }^{5,6}$ Since then, the direct current (dc) transport of surface-gated modulation-doped devices has been extensively studied, as reviewed by Beenakker and van Houten, ${ }^{7}$ but very little FIR spectroscopy of QPCs has been reported. FIR techniques have been used in

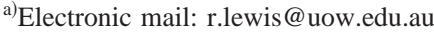

the characterization of quantum wires. ${ }^{8}$ Wyss et al. ${ }^{9}$ observed a FIR photoresponse in a QPC, which they attributed to heating effects. Janssen et al. ${ }^{10}$ claimed the first observation of a dc-voltage response to FIR radiation in a QPC. Theoretical investigations have also been carried out on these devices. ${ }^{11,12}$

The investigations to date, both experimental and theoretical, have largely ignored the effect of an applied magnetic field. At zero magnetic field the conductance of a QPC is quantized in units of $2 e^{2} / h$, reflecting the one-dimensional nature of the density of states. If a magnetic field is applied to the QPC the sub-bands are correctly described as magnetoelectric sub-bands, since both the electrostatic confinement and the magnetic confinement determine the density of states. As the field increases, the sub-band spacing increases and some sub-bands move above the Fermi energy. Consequently these sub-bands are depopulated, causing the magneto-conductance to oscillate. As the magnetic field is increased the number of conducting sub-bands tends towards the number of occupied Landau levels. Thus the transition from zero-field quantization to the quantum Hall effect is smooth. This phenomenon was first observed by van Wees et al. ${ }^{13}$

The FET structures on which the QPC investigated here

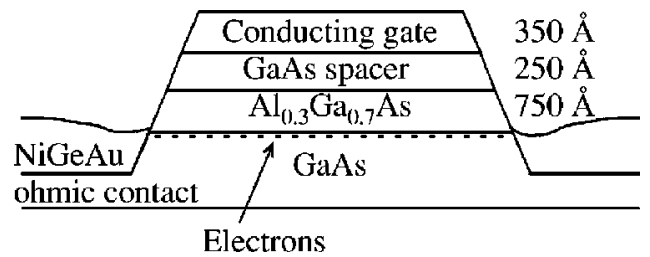

FIG. 1. Schematic view of the heterostructure on which the QPC was fabricated. 
TABLE I. Details of the FET structure on which the QPC was fabricated. The FET was grown on a GaAs substrate.

\begin{tabular}{lcc}
\hline \hline Layer & Composition & Thickness $(\AA)$ \\
\hline Top gate & $n^{+}$-GaAs & 350 \\
Spacer & $\mathrm{GaAs}$ & 250 \\
Barrier & $\mathrm{Al}_{0.3} \mathrm{Ga}_{0.7} \mathrm{As}$ & 750 \\
\hline \hline
\end{tabular}

was fabricated comprised a GaAs substrate, an $\mathrm{Al}_{0.3} \mathrm{Ga}_{0.7} \mathrm{As}$ barrier, a GaAs spacer layer, and a $n^{+}$-GaAs conducting top gate. A schematic view of the structure is given in Fig. 1 and details of the layers are listed in Table I. On biasing the top gate, a $2 \mathrm{DEG}$ forms at the AlGaAs/substrate interface. The electron sheet density, $n_{s}$, may be varied over the range $0.2-5 \times 10^{11} \mathrm{~cm}^{-2}$ by adjusting the top-gate bias. Etching produced a mesa of active area $100 \mu \mathrm{m}$ square. Contact was made to the 2DEG via NiAuGe self-aligned contacts. The process is described in detail elsewhere. ${ }^{14}$ To produce the QPC, side gates are formed by selective etching of the top gate in a pattern defined by electron beam lithography, as shown in Fig. 2(d) of Ref. 1.

The optical experiments reported here require a source of FIR and a means of detection. The FIR radiation was generated by an optically pumped molecular-gas $\operatorname{laser}^{15}$ and directed through an evacuated light-pipe to the sample which was situated in the bore of a superconducting magnet. The magnetic field, B, was parallel to the direction of light propagation, $\mathbf{k}$, and perpendicular to the FET layers (Faraday geometry). The FIR was detected by the photoconductive response of the device. The laser beam and the sample current were modulated at separate frequencies and the sample voltage was demodulated twice to recover the photosignal. The double-modulation technique was used to eliminate photovoltaic effects and to minimize noise in the data.

A distinctive photoresponse consisting of several peaks of varying intensity was observed in the QPC. These peaks coincide in magnetic field with minima in the Shubnikov-de Haas oscillations observed in transport data, as illustrated in Fig. 2. As the top-gate voltage is increased, and so the carrier density increased, the peaks systematically shift in field. This

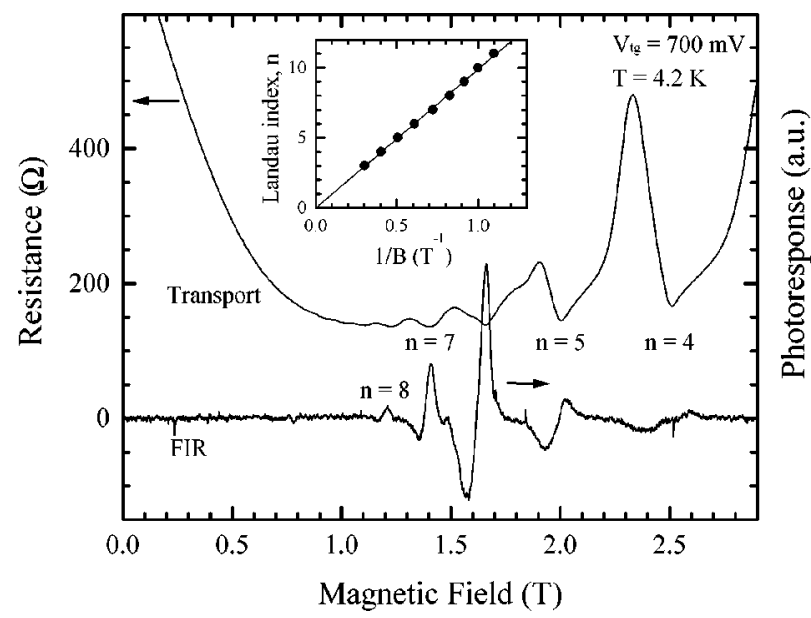

FIG. 2. FIR photoresponse for top-gate voltage $V_{\mathrm{tg}}=700 \mathrm{mV} ; \quad \lambda$ $=432 \mu \mathrm{m}$, and $T=4.2 \mathrm{~K}$. Transport data for the same top-gate voltage and temperature are included for reference. Inset: The positions of the magnetoresistance peaks in reciprocal magnetic field plotted against Landau index.

Downloaded 03 Sep 2008 to 130.130.37.13. Redistribution subject

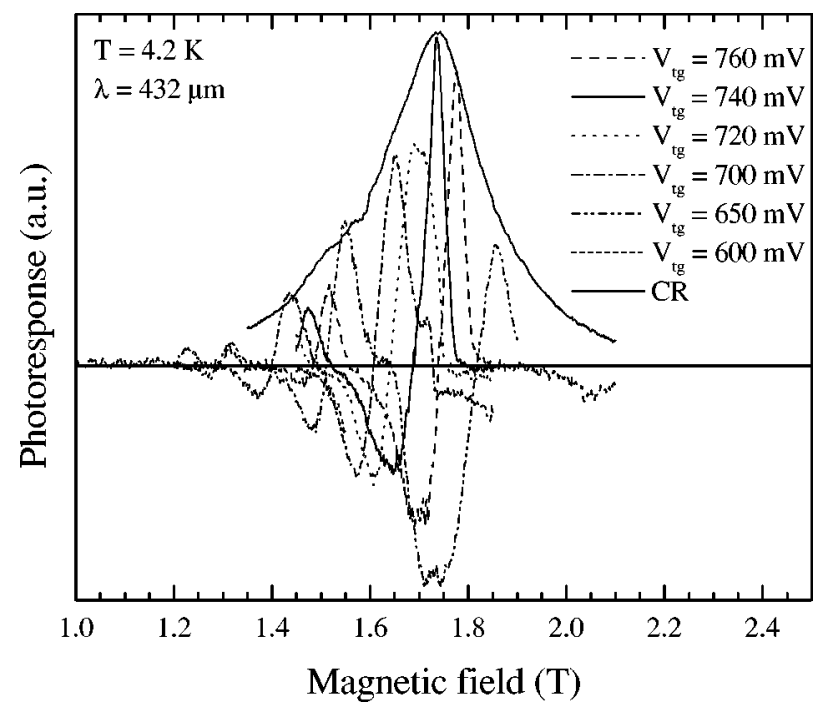

FIG. 3. FIR magneto-photoresponse for different top-gate voltages; $\lambda$ $=432 \mu \mathrm{m}, T=4.2 \mathrm{~K}$. The data fall inside an envelope centered on the $2 \mathrm{D}$ CR position.

shift matches the shift in Shubnikov-de Haas peaks with changing top-gate voltage.

The observed photoresponse moves in magnetic field as the laser wavelength is changed. The positions of the peaks change so that they remain within the region of the CR. The intensity of the peaks varies, with the strongest intensity feature (for constant laser power) at a field coincident with the 2D CR. If these data are plotted on the same vertical scale, as in Fig. 3, the intensities fall inside an envelope centered on the 2D CR. This envelope has the same shape as a 2D CR curve. The horizontal line in Fig. 3 is zero photoresponse.

This information taken together implies that the photoresponse is a resonant heating effect. From Fig. 2 it is clear that the photoinduced peaks coincide with dips in the transport. If the device is heated slightly, the transport structure decreases in amplitude, and subtracting data at two slightly different temperatures will result in an oscillatory structure such as that observed. In addition, the peaks move systematically with laser wavelength, and occur only in the region of the CR, implying that the effect is resonant with the cyclotron energy.

A similar resonant heating effect has been reported ${ }^{16}$ in InAs QWRs under free-electron-laser radiation at low powers. At high powers nonresonant heating effects are observed. ${ }^{16}$

In summary, a QPC fabricated on an undoped, top-gated FET structure exhibited a distinctive FIR magnetophotoresponse. Large oscillations periodic in inverse field were observed in photoconductivity. These oscillations depended sensitively on the top-gate voltage. By comparison with transport data from the same device and with CR measurements on the FET base material, it is deduced that the oscillations arise from resonant heating.

This work was supported in part by the Australian Research Council, the University of Wollongong, and the University of New South Wales. The authors thank the University of Canterbury for access to computing facilities.

AlP license or copyright; see http://apl.aip.org/apl/copyright.jsp 
${ }^{1}$ B. E. Kane, G. R. Facer, A. S. Dzurak, N. E. Lumpkin, R. G. Clark, L. N. Pfeiffer, and K. W. West, Appl. Phys. Lett. 72, 3506 (1998).

${ }^{2}$ P. M. Solomon, C. M. Knoedler, and S. L. Wright, IEEE Electron Device Lett. EDL-5, 379 (1984).

${ }^{3}$ G. R. Facer, B. E. Kane, A. S. Dzurak, R. J. Heron, N. E. Lumpkin, R. G. Clark, L. N. Pfeiffer, and K. W. West, Phys. Rev. B 59, 4622 (1999).

${ }^{4}$ R. J. Heron, R. A. Lewis, R. G. Clark, R. P. Starrett, B. E. Kane, G. R. Facer, N. E. Lumpkin, D. G. Rickel, L. N. Pfeiffer, and K. W. West, Physica B 256-258, 481 (1998).

${ }^{5}$ B. J. van Wees, H. van Houten, C. W. J. Beenakker, J. G. Williamson, L. P. Kouwenhoven, D. van der Marel, and C. T. Foxon, Phys. Rev. Lett. 60, 848 (1988).

${ }^{6}$ D. A. Wharam, T. J. Thornton, R. Newbury, M. Pepper, H. Ahmed, J. E. F. Frost, D. G. Hasko, D. C. Peacock, D. A. Ritchie, and G. A. C. Jones, J. Phys. C 21, L209 (1988).

${ }^{7}$ C. W. J. Beenakker and H. van Houten, in Solid State Physics, edited by H. Ehrenreich and D. Turnbull (Academic, New York, 1991), Vol. 44, pp. $1-228$.
${ }^{8}$ S. Wang, K. Yamanaka, K. Hirakawa, M. Noguchi, and T. Ikoma, Jpn. J. Appl. Phys., Part 2 35, L1249 (1996)

${ }^{9}$ R. Wyss, C. C. Eugster, J. A. del Alamo, and Q. Hu, Appl. Phys. Lett. 63, 1522 (1993)

${ }^{10}$ T. J. B. M. Janssen, J. C. Maan, J. Singleton, N. K. Patel, M. Pepper, J. E. F. Frost, D. A. Ritchie, and G. A. C. Jones, J. Phys.: Condens. Matter 6, L163 (1994).

${ }^{11}$ Q. Hu, Appl. Phys. Lett. 62, 837 (1993).

${ }^{12}$ F. A. Maaø and L. Y. Gorelik, Phys. Rev. B 53, 15885 (1996).

${ }^{13}$ B. J. van Wees, L. P. Kouwenhoven, H. van Houten, C. W. J. Beenakker, J. E. Mooij, C. T. Foxon, and J. J. Harris, Phys. Rev. B 38, 3625 (1988).

${ }^{14}$ B. E. Kane, L. N. Pfeiffer, and K. W. West, Appl. Phys. Lett. 67, 1262 (1995).

${ }^{15}$ Model FIRL 100; Edinburgh Instruments, Riccarton, Currie, Edinburgh, EH14 4AP, UK.

${ }^{16}$ J. Kono, Y. Nakamura, X. G. Peralta, J. Černe, S. J. Allen, Jr., H. Akiyama, H. Sakaki, T. Sugihara, S. Sasa, and M. Inoue, Superlattices Microstruct. 20, 383 (1996). 